[0212-7199 (2005) 22: 5; pp 207-208] ANALES DE MEDICINA INTERNA Copyright @ 2005 ARAN EDICIONES, S.L.

AN. MED. INTERNA (Madrid) Vol. 22, N. ${ }^{\circ}$ 5, pp. 207-208, 2005

\title{
Alteraciones pulmonares en los pacientes cirróticos: actualizaciones de un problema conocido
}

Calleja Panero JL, Albillos Martínez, A. Alteraciones pulmonares en los pacientes cirróticos. An Med Interna (Madrid) 2005; 22: $207-208$.

El síndrome hepatopulmonar (SHP) se caracteriza por una tríada que incluye enfermedad hepática, oxigenación arterial anormal y la presencia de dilataciones vasculares intrapulmonares (1).

La presencia de dilataciones precapilares, capilares y comunicaciones arteriovenosas empeoran la oxigenación arterial de los hematíes al incrementar la distancia entre los capilares sanguíneos y los alvéolos pulmonares, creando un shunt derecha-izquierda.

La prevalencia de este síndrome en los pacientes cirróticos varía entre el 4-32\% (2,3) dependiendo de la rigidez de los criterios valorados en los diferentes estudios.

La patogenia de la vasodilatación pulmonar no es completamente conocida aunque se ha implicado al óxido nítrico $(\mathrm{ON})$, potente vasodilatador, en su desarrollo (4-6). Sin embargo, no se conoce la fuente celular de $\mathrm{ON}$ en el pulmón ni los factores que regulan su producción. La mayor parte de los casos de SHP se han descrito en el contexto de una cirrosis hepática con hipertensión portal, pero se ha observado también en pacientes con trombosis portal sin cirrosis (7), e incluso asociadas a hepatitis aguda (8) o crónica (9) sin componente de hipertensión portal.

El diagnóstico de sospecha se basa en la detección de los pacientes que presenten alteraciones en la oxigenación arterial. El mejor método para evaluar el grado de oxigenación arterial no está claramente definido. La determinación del gradiente alvéolo-arterial de oxígeno $\left(\mathrm{AaO}_{2}\right)$, que también valora la presión parcial de monóxido de carbono $\left(\mathrm{PaCO}_{2}\right)$, es un método más fiable que la presión parcial de oxígeno $\left(\mathrm{PaO}_{2}\right)$, cuyo valor puede ser enmascarado por hiperventilación compensatoria y por el propio estado de circulación hiperdinámica que suele acompañar a estos pacientes (10).

El SHP se ha divido en tipo I y II. El tipo I se caracteriza por la presencia de dilataciones precapilares y capilares y por que responde a oxígeno al 100\%. El tipo II se asocia cambios vasculares más importantes, se acompaña de comunicaciones arteriovenosas que no responden a la terapia con oxígeno y cuyos parámetros gasometricos pueden mejorar si se embolizan esas malformaciones vasculares (11).

Lorenzo-Zúñiga et al, publican en este número de la revis- ta (12) un estudio prospectivo de la prevalencia de las alteraciones gasométricas en 50 pacientes cirróticos consecutivos que ingresan en su hospital. La alteración gasométrica más frecuente fue el aumento del $\mathrm{AaO}_{2}$, definido como mayor de $15 \mathrm{mmHg}$, presente en el $80 \%$ de los enfermos. Cuando se elevó el umbral hasta $20 \mathrm{mmHg}$, la prevalencia se redujo hasta el $62 \%$ de los pacientes. La hipoxemia y la hipocapnia se observaron en el 40 y del $46 \%$ de los pacientes, respectivamente, similar a lo publicado en otros estudios (13). De igual manera, la prevalencia del SHP definido con criterios más estrictos $\left(\mathrm{PaO}_{2}<70 \mathrm{~mm} \mathrm{Hg}\right.$ y $\left.\mathrm{AaO}_{2}>20 \mathrm{mmHg}\right)$ fue del $16 \%$ similar a la mayor parte de series publicadas $(2,14)$.

En este estudio se observó una correlación directa significativa entre el grado de alteración del $\mathrm{AaPO}_{2}$ y el de insuficiencia hepática definido por el estadio de Child-Pugh, siendo mayor el $\mathrm{AaPO}_{2}$ en los pacientes Child-Pugh B y C en comparación con el Child $\mathrm{A}$. Además en el análisis uni y multivariante las variables que se asocian a una mayor alteración del $\mathrm{AaO}_{2}$ son las determinantes del grado de insuficiencia hepática como los niveles plasmáticos de albúmina y el tiempo de protrombina.

El escaso número de pacientes en cada grupo no permite sacar conclusiones sobre la prevalencia de SHP según el grado de Child-Pugh. Se observó una frecuencia significativamente superior en pacientes con encefalopatía hepática, parámetro clásicamente relacionado también con el grado de insuficiencia hepática. Esta asociación entre frecuencia de SHP y gravedad de la enfermedad hepática ha sido recientemente descrita por Schenk et al (3) que observaron que la prevalencia de SHP fue casi doble en pacientes Child C en comparación con los Child A.

Pero estos hallazgos no son coincidentes con otros estudios que han descrito una mayor frecuencia y gravedad de SHP en pacientes A y B de Child $(14,15)$. Un estudio prospectivo que incluye a una cohorte de más de 200 cirróticos recientemente publicada (16) encuentra una prevalencia mayor en pacientes Child A (13\%) y Child B (11\%) que en pacientes Child C (1,4\%). Estas diferencias entre los diferentes estudios pueden atribuirse al escaso número de pacientes incluidos y al sesgo de selección. 
Una aportación original de este estudio es la descripción de una mayor alteración gasométrica en los pacientes que presentan una hepatitis alcohólica grave. Esto puede deberse al profundo deterioro de la función hepática que se produce en estos pacientes así como al empeoramiento de la circulación hiperdinámica frecuentemente asociado. Sería interesante evaluar la correlación entre el deterioro en los parámetros habitualmente utilizados en la valoración del estado hiperdinamico (gasto cardiaco, tensión arterial, resistencias vasculares periféricas) y los parámetros gasométricos.

Al no existir un tratamiento farmacológico que permita aliviar o corregir el síndrome hepatopulmonar, la única terapia definitiva es el trasplante hepático. Sin embargo la relación entre SHP y trasplante hepático es una relación conflictiva: por un lado se ha observado que la mayor parte de las anomalías en la oxigenación arterial revierten tras el trasplante (17). Pero por otro lado, la presencia de síndrome hepatopulmonar, y especialmente la hipoxemia grave empeora los resultados del trasplante y es una contraindicación relativa para su realización.

Del estudio de Lorenzo-Zúñiga et al (12) se desprende que el síndrome hepatopulmonar es un trastorno progresivo que empeora a medida que lo hace la enfermedad hepática y que es un factor independientemente asociado con la supervivencia en pacientes con cirrosis (3). De esta conclusión se obtienen dos reflexiones practicas: la importancia de su detección precoz para poder incluirles de manera temprana en la lista de candidatos a trasplante hepático. La segunda reflexión incluye la necesidad de evaluar la inclusión de estos parámetros pulmonares en los sistemas de priorización de la espera en la lista de trasplante. De esta manera, trasplantando cuando las alteraciones pulmonares son leves se reducirán las complicaciones asociadas.

\section{J. L. CALLEJA PANERO, A. ALBILLOS MARTÍNEZ}

Servicio de Gastroenterología. Hospital Universitario Clínica Puerta de Hierro. Universidad Autónoma de Madrid

${ }^{1}$ Hospital Ramón y Cajal. Universidad de Alcalá de Henares. Madrid

\section{Bibliografía}

1. Krowka MJ. Hepatopulmonary syndrome:recent literature and implications of liver transplantation. Liver Traspl 2000; 6: S1-S35

2. Schenk P, Fuhrmann V, Madl C, Funk G, Lehr S, Kandel O, Muller C. Hepatopulmonary syndrome; prevalence and predictive value of variuos cut offs for arterial oxygenation and their clinical consecuences. Gut 2002; 51: 853-59

3. Schenk P, Schoniger-Hekele M, Fuhrmann V, Madl C, Silberhumer G, Muller C. Pronostic significance of the hepatopulmonary syndrome in patients with cirrhosis. Gastroenterology 2003; 125: 1042-1052

4. Rolla G, Brussino L, Colagrande P. Exhaled nitric oxide and impaired oxygenation in cirrhotic patients before and after liver transplantation. Ann Intern Med 1998; 129: 375-8

5. Rolla G, Brussino L, Colagrande P, et al. Exheled nitric oxide and oxygenation abnormalities in hepatic cirrhosis. Hepatology 1997; 26: 84247

6. Rolla G, Bucca C, Brussino L. Methylene blue in the hepatopulmonary syndrome. N Engl J Med 1994; 331: 1098

7. Binay K, Sen S, Biswas PK, Sanyal R, Jumdar DM, Biswas J. hepatopulmonary syndrome in inferior vena cava obstruction responding to cavoplasty. Gastroenterology 2000; 118: 192-96

8. Regev A, Yeshurum M, Rodríguez M, Sagie A, Neff EW, Molina EG, et al. Transient hepatopulmonary syndrome in a patient with acute hepatitis A. J Virsl Hepatol 2001: 8 (1) 83-6

9. Teuber G, Teupe C, Dietrich C, Caspary W, Buhl R, Zeuzem S. Pulmonary disfunction in non cirrhotic patients with chronic viral hepatitis. Eur J Intern Med 2002: 13: 311-18

10. Lima BLG, Franca AVC, Pazin-Filho A, Araújo WM, Martínez JAB, Maciel BC, et al. Frecuency, clinical characteristics and respiratory parameters of hepatopulmonary syndrome. Mayo Clin Proc 2004; 79 $42-48$

11. Krowka MJ, Cortese DA. Hepatopulmonary syndrome: classification by arterial oxygenation and pulmonary angiographic appearance. Chest 1992; 102; S124

12. Lorenzo-Zúñiga V, Álvarez MA, Planas R, Morillas R, Doménech E, Cabré E, et al. Alteraciones gasométricas en pacientes cirróticos hospitalizados. An Med Interna (Madrid) 2005; 22: 209-212.

13. Moller S, Hillings J, Chirstiensen E. Arterial Hypoxemia in cirrosis; fac. or fiction Gut 1998; 42: 868-874

14. Abrams GA, Jaffe CC, Hoffer PB, Binder HJ, Fallon MB. Diagnostic utility of contrast echocardiography and lung perfusion scan in patients with hepatopulmonary syndrome. Gastroenterology 1995; 109; 1283 1288

15. Krowka M, Wiseman G, Burnett O, Spivey J, Thernau T, Porayko M, et al. hepatopulmonary syndrome: a prospective study of relationships between severity of liver disease, $\mathrm{PaO} 2$ response to $100 \%$ oxygen and brain uptake after 99m Tc MAA lung scanning. Chest 2000; 118: 615-24

16. Abrams GA, Sanders MK, Fallon MB. Utility of pulse oxymetry in the detection of arterial hypoxemia in liver transplant candidates. Liver Trasplant 2002; 8: 391-96

17. Collison EA, Nourmand H, Fraiman MH. Retrospective analysis of the results of liver trasplantation for adults with severe hepatopulmonary syndrome. Liver Traspl 2002; 8: 925-931 